\title{
ELCS in Ice: Cryo-electron Microscopy of Nuclear Envelope Limited Chromatin Sheets
}

UNE UNIVERSITY OF

1. WHAT ARE ELCS? Nuclear Envelope-Limited Chromatin Sheets (ELCS) form during excessive interphase nuclear envelope growth in a variety of cells. ELCS appear as extended sheets within the cytoplasm connecting
distant nuclear lobes. Cross-section stained images of ELCS, viewed by transmission electron microscopy, resemble a distant nuclear lobes. Cross-section stained images of ELCS, viewed by transmission electron microscopy, res
sandwich of apposed nuclear envelopes separated by $\sim 30 \mathrm{~nm}$, containing a layer of ordered chromatin fibers.

\section{EM PROCEDURES} fixation/dehydration/plastic embedding/sectioning and staining; 2) high-pressure freezing/freeze substitution into cells were treated with retinoic acid (4 days) to induce granulopoiesis, growth of nuclear envelope membranes, formation-6o/S4) nuclei and ELCS.

\section{COMPARISON OF DIFFERENT PROCEDURES}
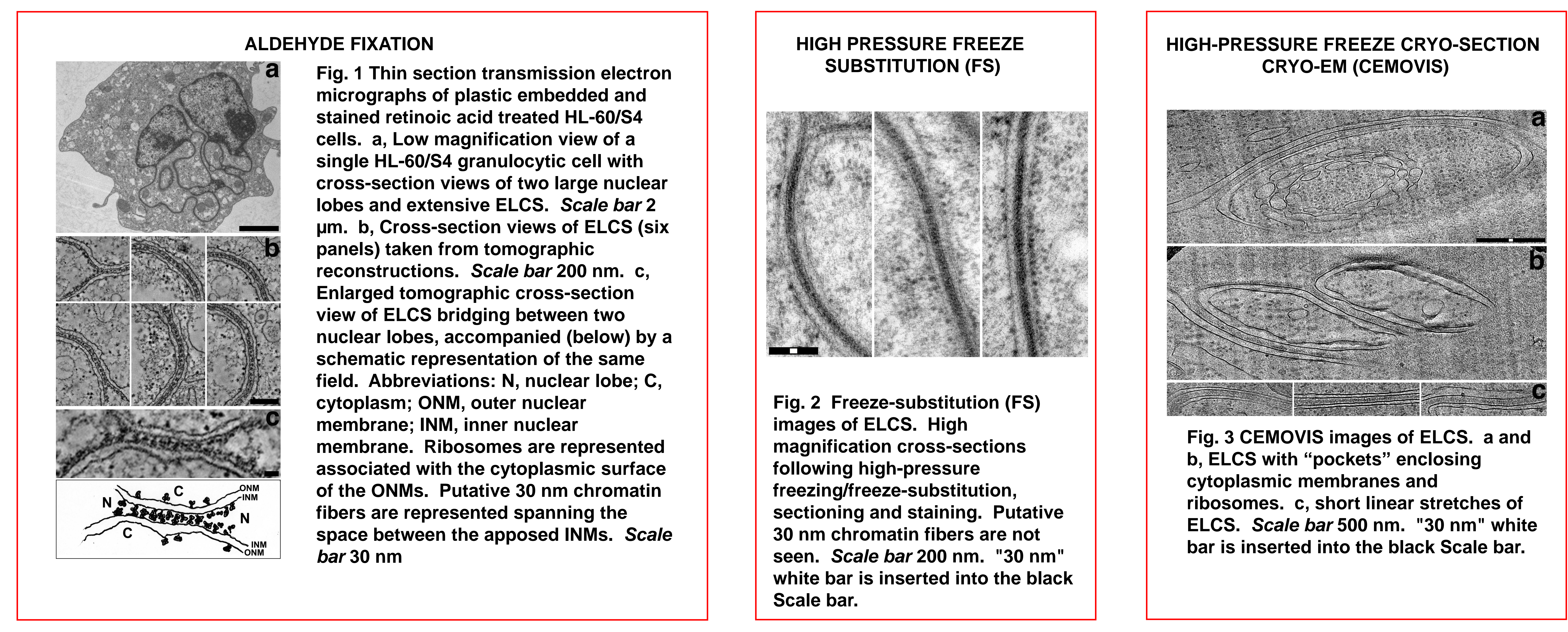

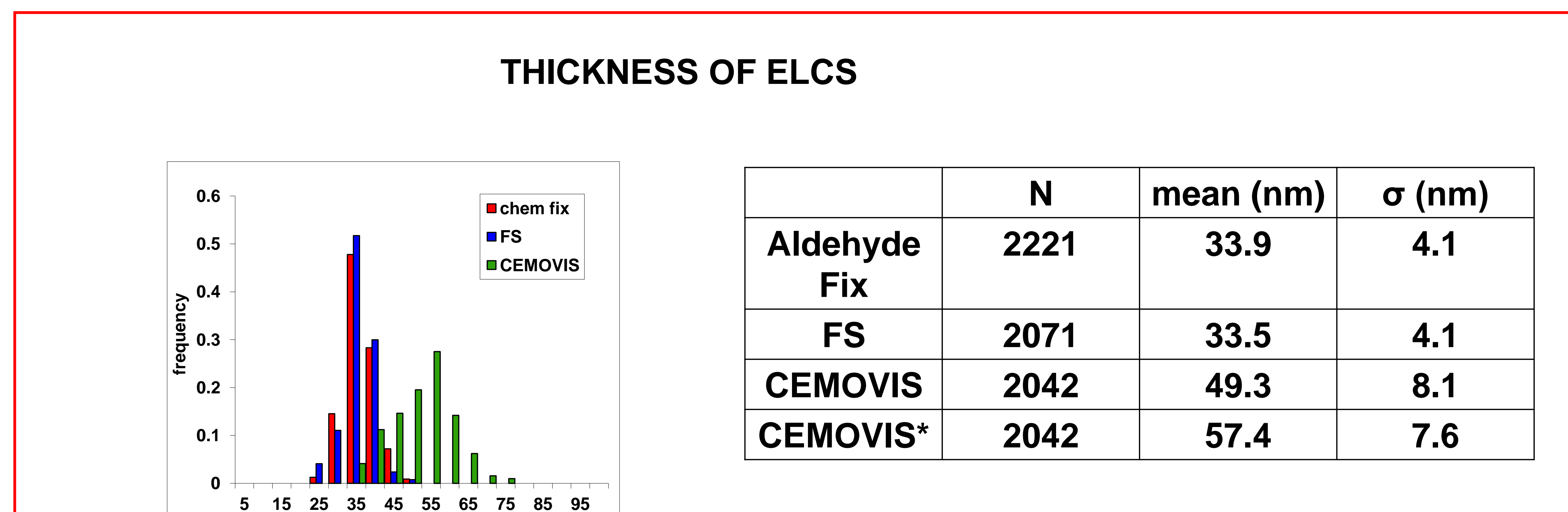

* Extrapolated uncompressed distance Table 1 Distance between apposed inner
nuclear membranes of ELCS. This table displays the measurement statistics, measurements $(\mathrm{N})$, and the mean $(\mathrm{nm})$ and standard deviations $(\sigma)$ of the distances between the inner nuclear membranes, comparing three sample

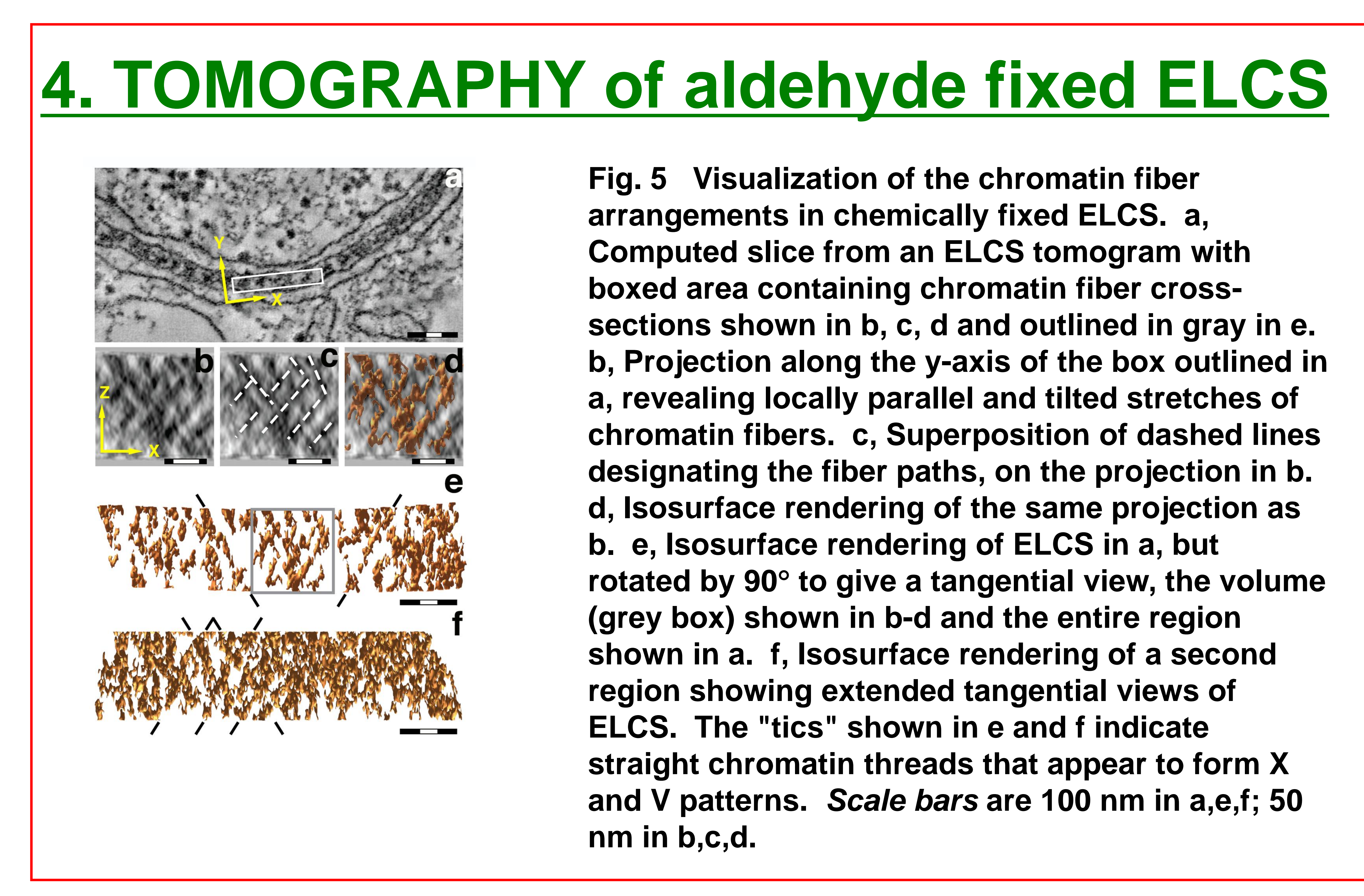

\section{CONCLUSIONS}

-ELCS exist in vivo. They are not an artifact of fixation.

-ELCS in ice are thicker than after dehydration and embedding in plastic.

-EM tomography of aldehyde fixed cells supports that the putative "30 nm fiber" in

ELCS are composed of two overlapping layers of parallel "10 $\mathrm{nm}$ fibers".

\section{MODEL}

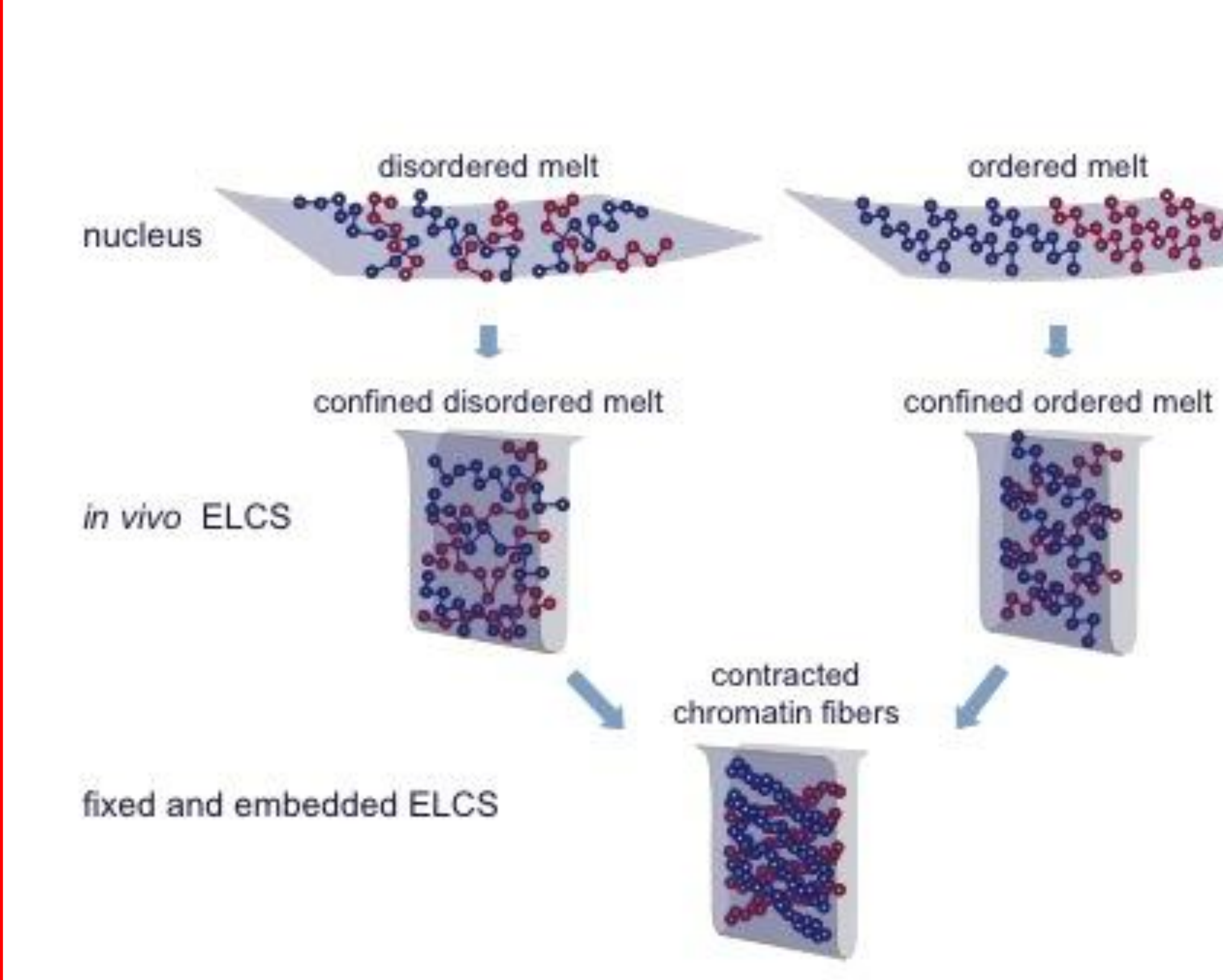

Fig. 6 Modeling chromatin fiber folding Schemes demonstation. how nuclear envelope associated $\sim 10 \mathrm{~nm}$ chromatin ("ordered mel" or "disordered melt") $30 \mathrm{~nm}$ fibers in aldehydo fixed, dehydrated, embedded and sectioned ELCS, as visualized by lomography.

\section{RECENTLY PUBLISHED in CHROMOSOMA 123 303-312, (2014)}

Cell Biology and Biophysics Unit, European Molecular Biology Laboratory, Heidelberg, Germany, eltsov@embl.de 2. Neurophysiology \& New Microscopies Laboratory, INSERM U603 - CNRS UMR 8154, Paris, France, sosnovski.sergey@gmail.com
3. University of New England, College of Pharmacy, Portland, ME, USA aolins@une.edu dolins@Qne.edu

\section{Acknowledgements}

The authors express their appreciation to the European Molecular Biology Laboratory (EMBL, Heidelberg), the German Cancer Research Center (DKFZ, Heidelberg) and the University of New England (Portland, Maine) for their support and who generously opened their lab. We particularly wish to thank Peter Lichter, Harald Herrmann and Jorg Langowski (DKFZ), Andreas Hoenger (Boulder, CO) and Rachel Santarella-Mellwige (EMBL) The Boulder Laboratory for to 3 -D Electron Micros and of Cells is supported by the National Center for Research Resources, NIH. 